\title{
THE JOINT TRAINING SYSTEM: A PILLAR OF JOINT READINESS AND AN AUTOMATION CHALLENGE
}

\author{
Joseph C. Barto, III \\ Lieutenant Colonel, United States Army \\ Joint Warfighting Center \\ Fort Monroe, VA 23651
}

\author{
Patrick D. Clark \\ Commander, United States Navy \\ Joint Warfighting Center \\ Fort Monroe, VA 23651
}

\begin{abstract}
The Joint Training System (JTS) (Figure 1) defines a multi-step approach to identify training requirements, plan, execute, and assess joint training events. First, and foremost, the JTS is focused on warfighting. "The specific objective is to develop a joint training program that bolsters the combatant commanders' ability to execute the National Military Strategy while simultaneously maintaining high readiness of our forces as a pre-requisite to deterring aggression and responding to crisis. The desired end state is the improved readiness of joint forces, a training and exercise strategy better aligned with the National Military Strategy, improved interoperability, and a more stable process for optimizing the application of scarce resources (dollars, forces, time)." Successful implementation of the JTS depends upon automating the system's processes so that the development, sharing, and reporting of training information and
\end{abstract}

products is conducted as efficiently and effectively as possible.

\section{INTRODUCTION}

Readiness is the right people, properly equipped, trained as a team capable of fighting and winning our nation's wars. That formula with its fundamental dependent relationships relates the commander's age old problem. "Given my assigned missions how do I best man, equip, and train my forces to successfully accomplish those missions; and if I have done everything I can and still determine that I can not accomplish the mission how do I tell my boss in terms so that he can help me." Consequently; training proficiency, equipment availability, and personnel resources are the three pillars of joint readiness. These

\section{The Joint Training System}

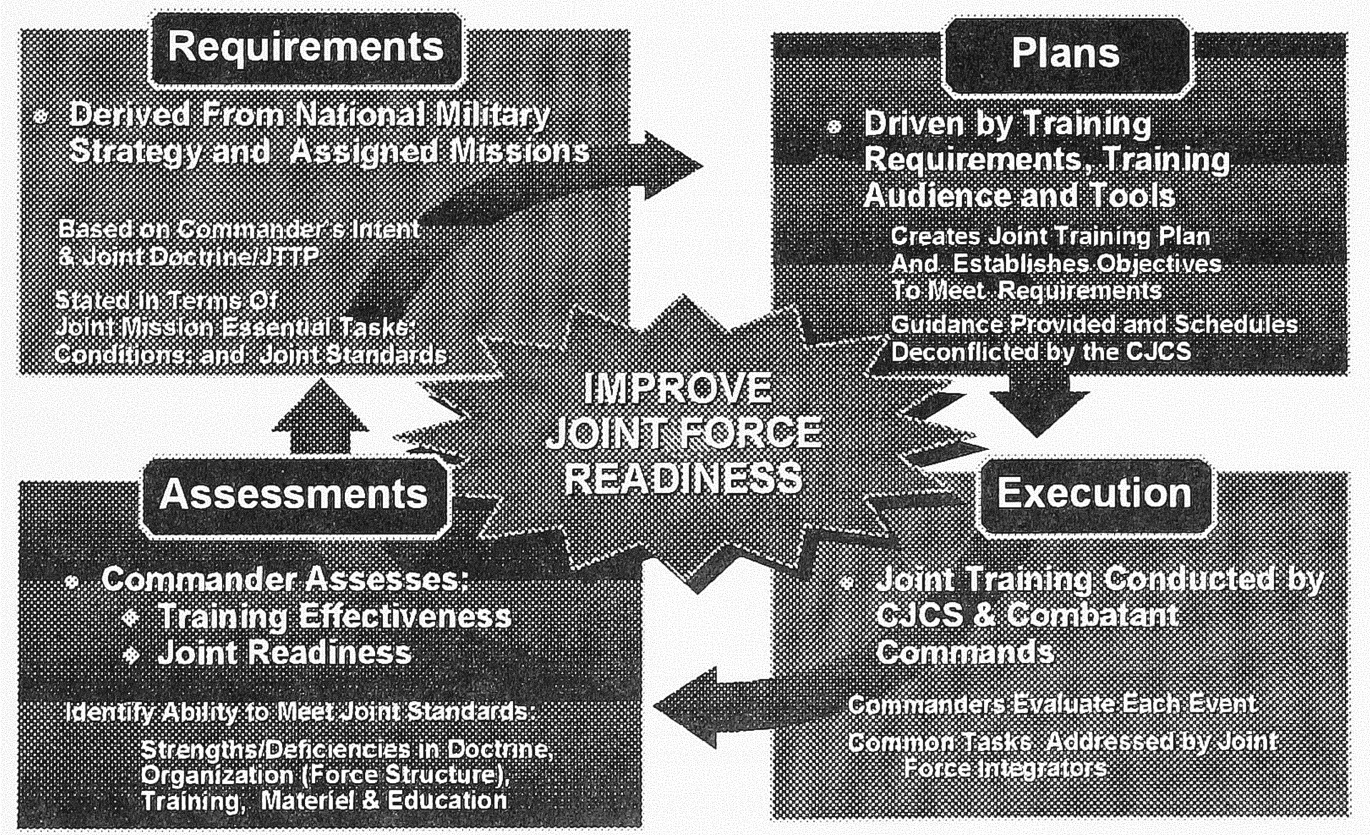

Figure 1: The Joint Training System 
three pillars directly affect the creation and maintenance of a trained and ready force able to perform assigned missions.

Under Title $\mathrm{X}$ of the United States Code, the combatant commanders (e.g. the Commanders and Chiefs of US European Command, US Atlantic Command, US Central Command, US Southern Command, US Pacific Command, US Transportation Command, US Strategic Command, US Special Operations Command, and US Space Command) are responsible for assessing their own joint training proficiency. The Goldwater Nichols Act directed the Chairman, Joint Chiefs of Staff (CJCS) to develop a uniform system to assess the preparedness of the combatant commanders to carry out assigned missions. The emerging JTS as directed in the Joint Training Master Plan (JTMP) links joint training to joint readiness. The Universal Joint Task List (UJTL), developing Joint Mission Essential Task Lists (JMETLs), preparing task-based joint training plans, executing and evaluating task-based joint training, and assessing training proficiency based upon demonstrated performance establish a uniform system for joint training readiness assessment; and more importantly, a methodology to correct those deficiencies and validate the solutions within the same task-based training system.

Using this system, the combatant command, using JMET derived training objectives for individual training events conduct training evaluations tied directly to those training objectives. The UJTL provides a common framework to describe the full range of tasks which may be accomplished at the Strategic National, Strategic Theater, Operational levels of war and the primary purview of the Joint Force Commander and provides a link to the Service Tactical Task Lists. UJTL derived JMETLs are a comprehensive and authoritative standard with approved tasks, conditions, and standards. Since the JMETL reflects those essential tasks which must be accomplished in order to accon:plish the mission, the training proficiency evaluation of those JMETs reflects and provides insights to the command's ability to accomplish the assigned missions. This linkage closes the joint training system cycle--the command's readiness to accomplish its missions.

\section{Joint Training System: Process and Products}

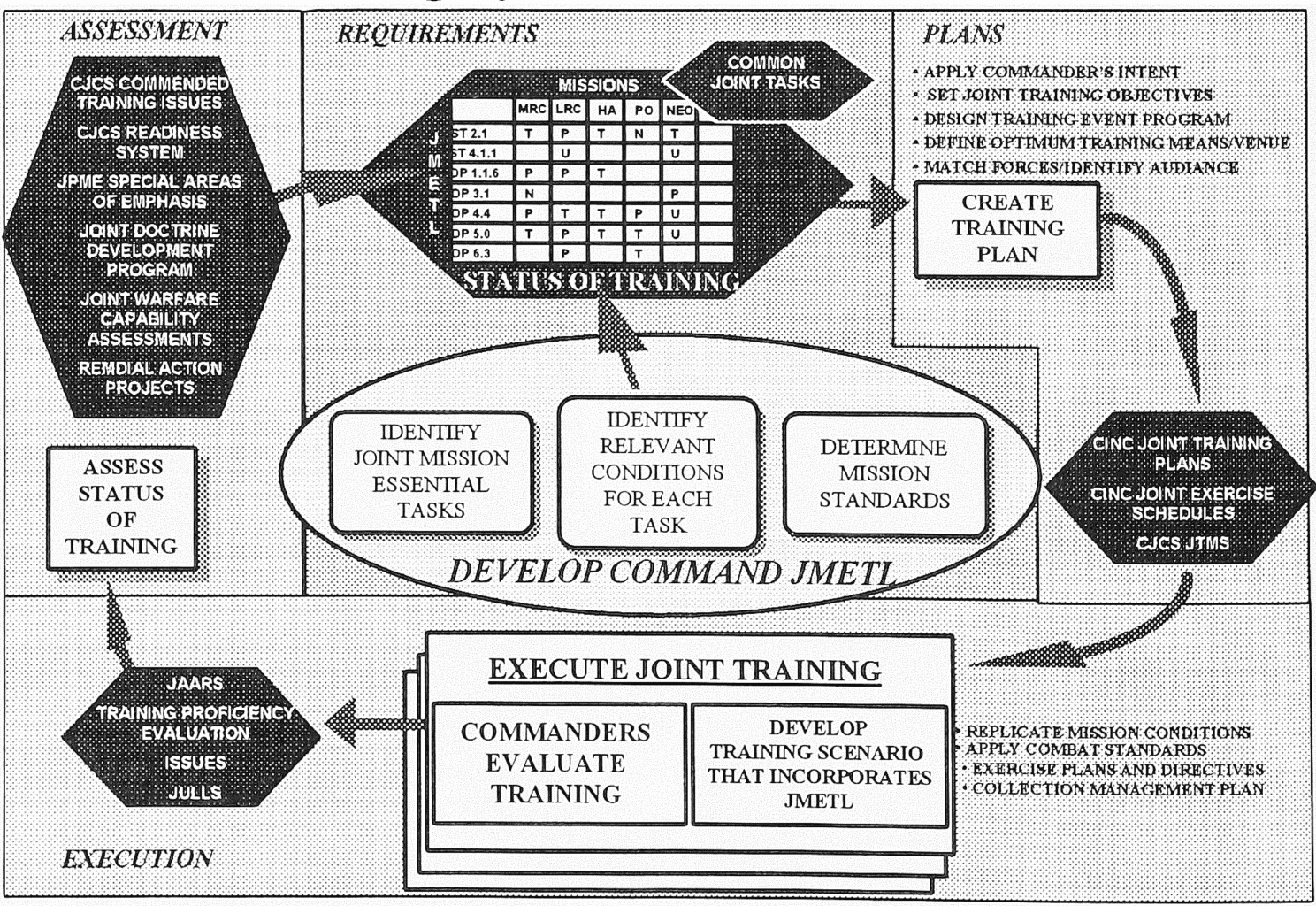

Figure 2. Joint Training System: Process and Products 
2 The Joint Training System Processes and Products

There are specific processes and products associated with each phase of the JTS (see Figure 2). The four phases of the JTS are Requirements, Plans, Execution, and Assessment.

\subsection{Phase I: Requirements.}

The purpose of this phase is to install the methodology and tools to translate strategy to missions to tasks. The mission-to-task JTS revolves around clear statements of joint requirements. This phase uses major inputs including: analysis of operation plan (OPLAN, CONPLAN, functional plan) missions resulting from Joint Strategic Capabilities Plan (JSCP) planning tasks, joint doctrine, and the Universal

\subsection{Phase II: Planning.}

Once command requirements are approved and training readiness assessments are considered, joint training plans and exercise schedules are developed to address the JMETL requirements. The objective is to incrementally install tasks, conditions and standards into joint training and exercise programs. The products of this phase are the CINC Joint Training Plans, CINC Joint Exercise and Training Schedules, and the CJCS Joint Training Master Schedule.

\subsection{Phase III: Execution}

The actual conduct and evaluation of joint and multinational training events and the support infrastructure to support a joint training event are the focus of the execution phase. Both collective joint training events and individual joint training events contribute to the personnel "train up" process in preparation for joint operations. Within this phase, discrete joint training exercises and events are planned, prepared, executed, and evaluated. Moreover, standardized training development tools and automated products assist trainers in executing JMETL-based training events. As part of the execution phase, commanders are responsible for systematically evaluating each training exercise or event to determine the level of training proficiency attained
Joint Task List (UJTL) as an interoperability tool.

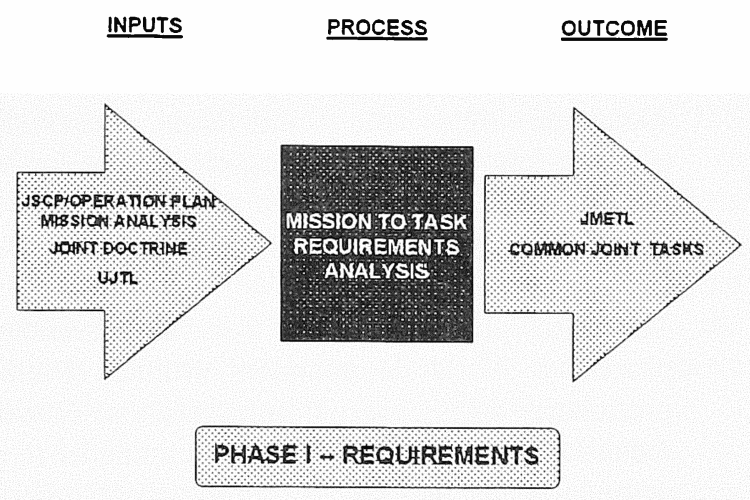

The end products are each combatant command's JMETL, and a multi-command list of common joint tasks.

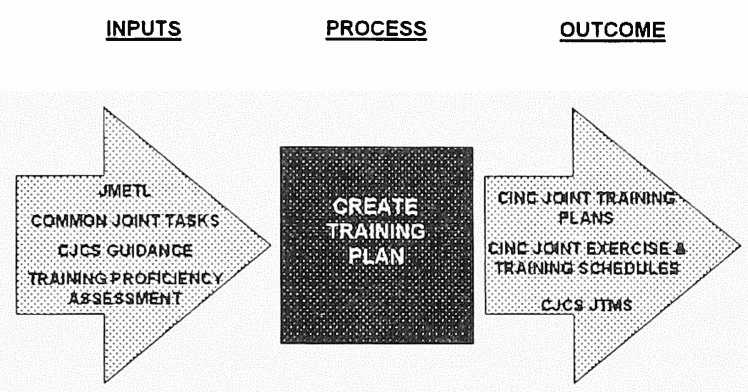

PHASE 11 - PLARILHNG

for each JMET-derived training objective. Evaluation is an internal command responsibility, intended to determine whether specific training objectives were met. In addition to the actual training received, the products of this phase are the Joint After Action Report (JAAR) task proficiency observations, command training proficiency evaluation, Joint Universal Lessons Learned (JULs) and Issues.

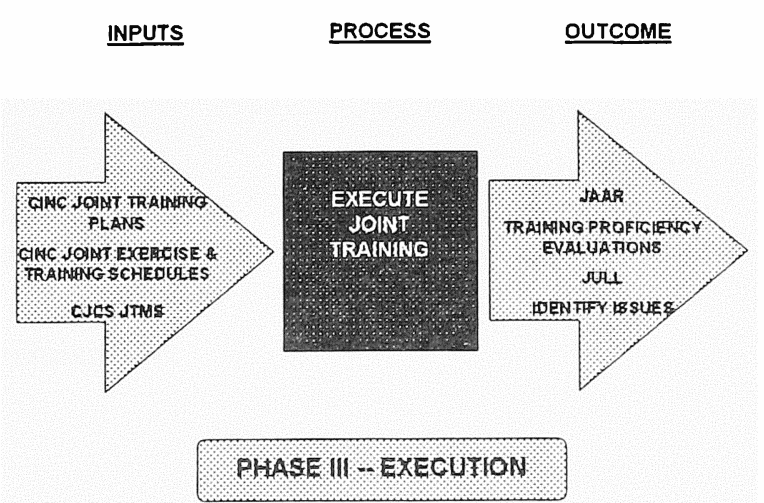


Ultimately, joint training depends on the efficient and effective execution of joint exercises and training events. Most of these activities fall under the CJCS Exercise Program consisting of those activities sponsored by the CJCS and those sponsored by the individual combatant commanders. These two categories can be further

\subsection{Phase IV: Assessment.}

The final phase of the JTS is the assessment process. While commanders evaluate training proficiency during every training exercise or event, the assessment phase allows the commander to use aggregated results from all training events to judge their commands overall mission capability. Assessments synthesize multiple training event evaluations with the commander's assessment of JMET proficiency. The assessment phase completes the joint training cycle. The products of this assessment serve to inform future training plans or, when high value issues are raised, near term training plans may require adjustment to focus on those critical shortcomings or

\section{Uses of Joint Training Assessment Information.}

The results of the commander's assessment dictates one or more of the following corrective actions:

\subsection{Adjust the current Joint Training Plan:}

If during the evaluation of individual training objectives during joint training events a shortcoming or deficiency is deemed immediately critical to mission accomplishment then the commander may elect to immediately revise training plans correcting that identified task shortcoming or deficiency. Commanders should understand that this decision may have drastic short term impacts on training events that are already well into the planning or preparation stages of the execution phase.

\subsection{Inform future Joint Training Plans:}

Training proficiency assessments are a key and essential portion of the joint training system. All things being equal commanders should focus their training resources and efforts on JMETL tasks assessed U (Untrained), P (Partially Trained), or N (Not deficiencies. This snapshot of a command's proficiency in accomplishing JMETs directly reflects the command's ability to perform assigned missions. Therefore, this assessment may be reported out by the command as input to CJCS and combatant command readiness reporting systems. Finally, systemic Issues requiring resolution outside the purview of the organization are defined, analyzed, corrected and returned to the joint community for validation.

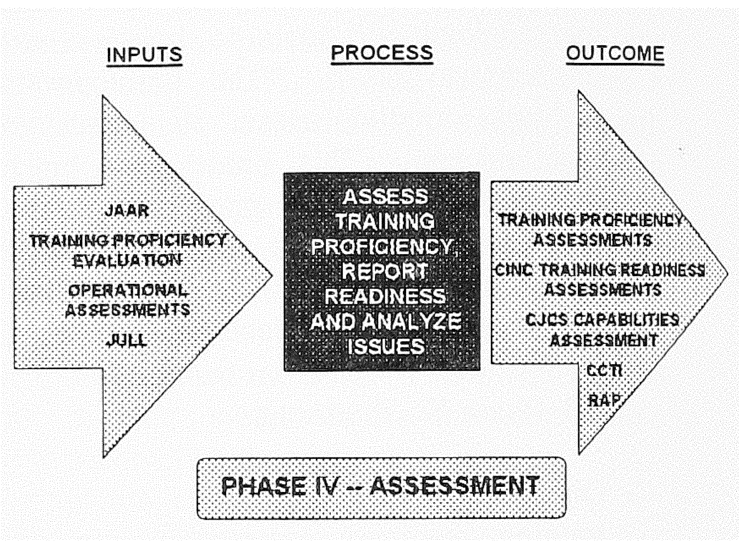

Observed). Obviously, an assessment of $\mathrm{T}$ (Trained) in any given task or JMET indicates full capability to perform that JMETL based task, under established conditions, to the desired standard. $T$ (Trained) is the goal. Theoretically, if the command is Trained in all the JMETs associated with a specific mission then the command can report that they are READY to perform that mission based upon demonstrated performance.

\subsection{Report out Readiness to perform assigned missions.}

Reports outside the command may come in many formats or purviews. All reporting is a request for assistance and not an admission of guilt or performance impotence. One format is the Joint Monthly Readiness Report (JMRR). For the MRC scenarios addressed in the JMRR, a joint training assessment is required to the CJCS as well as resource status in terms of personnel and equipment. This same information may be reported out to other agencies requesting status reports because it is based on demonstrated training proficiency against established 
standards. The Joint Warfighting Capabilities Assessment (JWCA) and the Remedial Action Projects (RAP) Program are two examples.

\subsection{Report Out Issues.}

Many identified deficiencies are addressed within the commands internal procedural or resource capabilities. However, those deficiencies outside the command's purview to correct are reported outside the command along with recommended corrections. The primary method to communicate deficiencies or shortcomings both within the command and to external agencies is through the Doctrine, Organization, Training, Materiel, Education (DOTME) construct. This analytical methodology allows for a comprehensive analysis to be conducted which considers all aspects of an issue. The result is a complete, coordinated and analyzed product leading the command and/or the joint community to comprehensive issue resolution. For example, if the organizational structure is changed; then the way that organization is employed doctrinally, trained, equipped, and its personnel educated is effected.
Over the next two years the CJCS has directed the full implementation of the JTS. Clearly, the JTS is an information intense system whose efficiency is largely dependent upon the rapid exchange and sharing information. Additionally, the installation of the JTS and its Automation Tools must not be so administratively burdensome that if fails simply from the weight of its complexity and manpower requirements. Finally, the automation effort must account for the needs of the user and focus on the products required and the allow for quick, reliable electronic communications around the world.

As the JTS processes are being validated, the automation effort is beginning under a program entitled the Joint Event Training Tool (JETT). (Figure 3) As the Program Management structure is organized and software integration and development occurs there are several key points to keep in mind.

4.1 Settle on a Program Structure and agree early on specific responsibilities. JETT has a Steering Group, a Configuration Control Board, a Program Manager, and an Executive Agent with specific technical expertise.

\section{The Automation Challenge.}

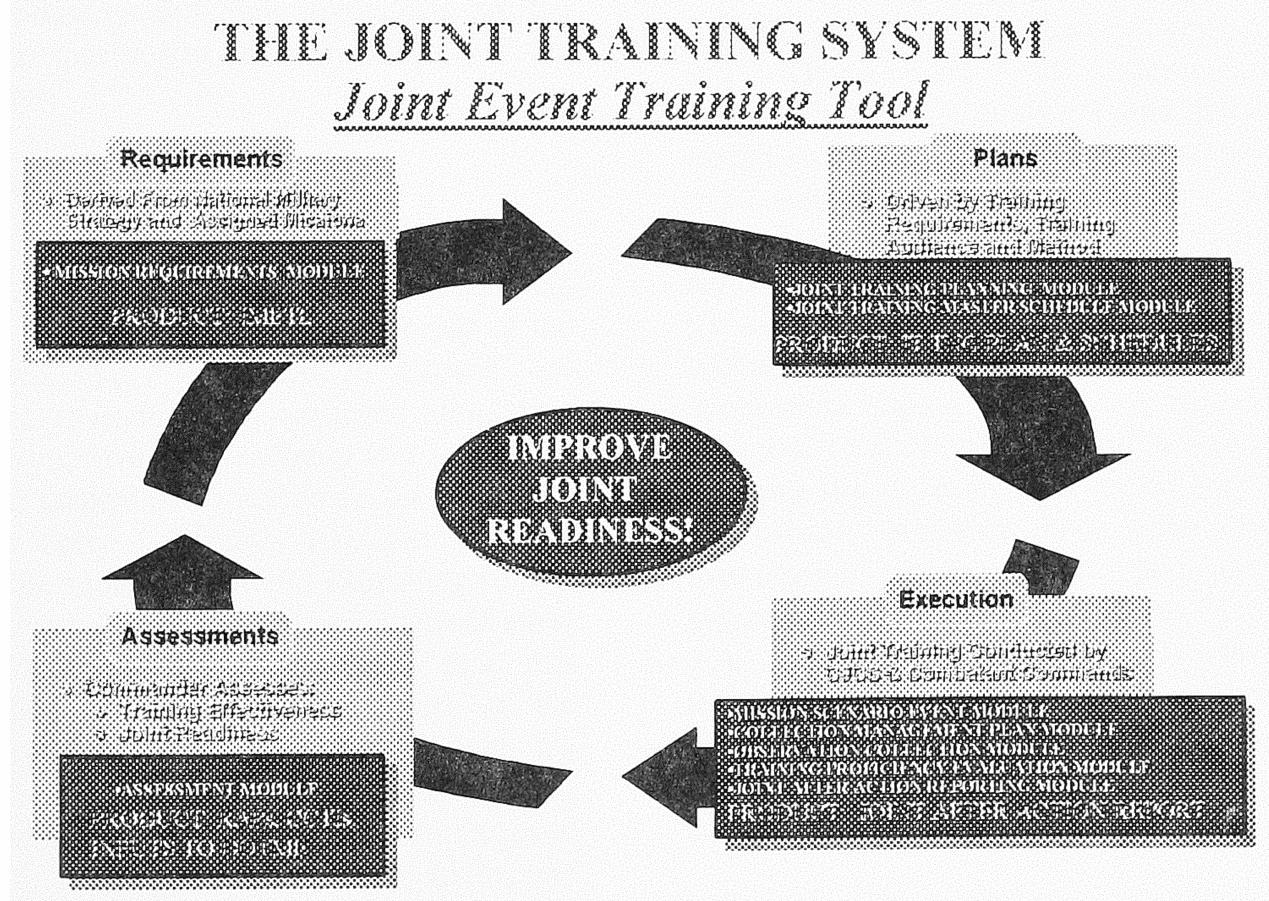

Figure 3: Joint Event Training Tool 
4.2 Ensure a thorough understanding of the process before building automation software. The functionality must be fully developed before considering software alternatives. Designate a specific effort to focus, capture and update the functional requirements document. An unclear understanding of the required functionality makes every piece of software look great.

4.3 Agree to a technical approach early and hold the line. DoD has many and varied rules and regulations applying to software and software development. Get an expert and put them on the CCB.

4.4 Constantly stay aware of the user resources required. Education may be the most expensive resource and will dictate the success of the implementation plan. Ensure the entire life cycle is considered to include initial training, distribution, and life cycle costs. Putting some disks in the mail with installation instructions is not a fielding plan.

4.5 Develop an implementation and public relations campaign up front. Bring the customers in early and often and make them part of the developmental team. Create a sense of ownership among your users. Use of existing software that may have been developed locally helps. Do not discount anything a user brings to the table without a full evaluation.

\section{REFERENCES:}

Chairman Joint Chiefs of Staff Instruction 3500.01, Joint Training Policy, dtd 21 November 1994

Chairman Joint Chiefs of Staff Instruction 3500.02A Joint Training Master Plan 1998, dtd 8 December 1995

Chairman Joint Chiefs of Staff Instruction 3500.03 Joint Training Manual, dtd 1 June 1996

\section{AUTHOR BIOGRAPHIES}

JOSEPH C. BARTO, III is a Lieutenant Colonel in the United States Army currently assigned to the Joint Warfighting Center at Fort Monroe, Virginia. He is holds a B.S. from the United States Military Academy at West Point, NY and a MPA from James Madison University at Harrisonburg, VA.

PATRICK D. CLARK is a commander in the United States Navy currently assigned to the Joint Warfighting Center at Fort Monroe, VA. He holds a B.A. from the University of Wisconsin and a M.A. from the Naval War College at Newport News, RI. 